

\title{
Ethyl 2-(Diisopropoxyphosphoryl)-2H-azirine-3-carboxylate: Reactions with Nucleophilic 1,3-Dienes
}

\author{
M. José Alves, ${ }^{* a}$ Américo Lemos, ${ }^{\text {b }}$ José Enrique Rodriguez-Borges, ${ }^{c}$ Xerardo García-Mera, ${ }^{\mathrm{d}}$ A. Gil Fortes ${ }^{\mathrm{a}}$ \\ a Departamento de Química, Universidade do Minho, Campus de Gualtar, 4710-057 Braga, Portugal \\ Fax +351(253)678983; E-mail: mja@quimica.uminho.pt \\ b Departamento de Química, Bioquímica e Farmácia, Universidade do Algarve, Campus de Gambelas, 8005-137 Faro, Portugal \\ Fax +351(289)819403; E-mail: alemos@ualg.pt \\ c CIQ, Departamento de Química, Faculdade de Ciências, Universidade do Porto, Rua do Campo Alegre, 687, 4169-007 Porto, Portugal \\ d Departamento de Química Orgánica, Facultade de Farmacia, Universidade de Santiago de Compostela, 15782 Santiago de Compostela, \\ Spain
}

Received 24 March 2009; revised 18 May 2009

\begin{abstract}
Ethyl 2-(diisopropoxyphosphoryl)- $2 H$-azirine-3-carboxylate, the first example of an azirine bearing simultaneously ester and phosphonate groups was generated in situ and reacted with a number of 1,3-dienes. Cycloadducts or their ensuing rearranged products were isolated in moderate yields.
\end{abstract}

Key words: $2 H$-azirines, aza-Diels-Alder cycloaddition, dienophiles, phosphonates

$2 \mathrm{H}$-Azirines have generated a great deal of interest due to their versatility as building blocks in the synthesis of important classes of heterocyclic compounds, ${ }^{1,2}$ and amino acids. ${ }^{3} 2 \mathrm{H}$-Azirines carrying ester groups are especially important not only due to their structural similarity to naturally occurring azirines with biological activity, like azirinomycin ${ }^{4}$ and (-)-( $R$-dysidazirine antibiotics, ${ }^{5}$ but also for being excellent precursors in the synthesis of $\alpha^{-6}$ and $\beta$-amino acid $^{7}$ derivatives. Azirines with $\mathrm{C}=\mathrm{O}, \mathrm{P}=\mathrm{O}$ or heteroaromatic groups conjugated with the $\mathrm{C}=\mathrm{N}$ bond, are effective dienophile partners ${ }^{8-10}$ in Diels-Alder cycloadditions, producing bicyclic and tricyclic compounds. $2 \mathrm{H}$-Azirines devoid of electron-withdrawing groups only react with specially activated dienes such as 1,3-diphenylisobenzofuran in refluxing toluene ${ }^{11 \mathrm{a}, \mathrm{b}}$ or under Lewis acid catalysis. ${ }^{11 \mathrm{c}, \mathrm{d}}$

Excitatory amino acids are the most common neurotransmitters in the mammalian central nervous system thus their receptors have been exploited in the treatment of several pathological conditions affecting the brain, such as Parkinson's and Alzheimer's diseases. ${ }^{12}(S)$-2-Amino- 3-phosphonopropanoic acid [(S)-AP-3, 1, Figure 1] is known to be a modulator for the $N$-methyl-D-aspartate (NMDA) receptor site.<smiles>NC(CP(=O)(O)O)C(=O)O</smiles>

Figure 1 (S)-2-Amino-3-phosphonopropanoic acid

In connection with our work on $2 H$-azirines, we envisaged that 2-(dialkoxyphosphoryl)- $2 \mathrm{H}$-azirine-3-carboxylates would be excellent dienophiles for Diels-Alder cycloadditions, introducing simultaneously the biologically important phosphonate group ${ }^{13}$ into cycloadducts. This class of compounds has not been previously synthesized, despite of being closely related to $(S)$-AP-3 1 .

This paper reports the unprecedented generation of ethyl 2-(diisopropoxyphosphoryl)-2H-azirine-3-carboxylate (5) and its interception by a number of electron-rich buta-1,3dienes producing mono-, di-, and tricyclic aziridines, carrying the $\alpha$-amino- $\beta$-phosphonate carboxylate moiety.

The oxime 3 was obtained from ethyl bromopyruvate oxime (2) ${ }^{14}$ and triisopropyl phosphite. Its treatment with tosyl chloride in the presence of sodium carbonate led to $\beta$-phosphonic tosyloxime ester 4 (Scheme 1). The corresponding $2 \mathrm{H}$-azirine $\mathbf{5}$ was obtained under Neber conditions, but could not be isolated from the reaction medium. Although monofunctional $2 H$-azirine-2-phosphonates ${ }^{15}$

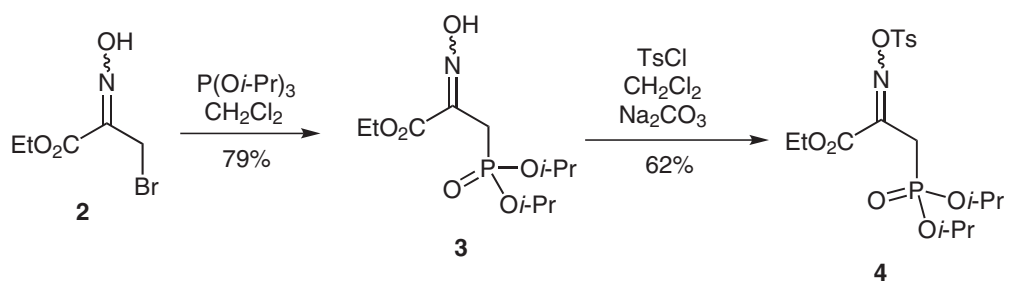

Scheme 1 Preparation of $\beta$-phosphonic tosyloxime ester

SYNTHESIS 2009, No. 19, pp 3263-3266

Advanced online publication: 21.08.2009

DOI: 10.1055/s-0029-1216945; Art ID: T06709SS

(c) Georg Thieme Verlag Stuttgart · New York 
have been produced and isolated before under similar reaction conditions, manipulation of the reaction mixture in the present case, however, led to decomposition, according to ${ }^{1} \mathrm{H}$ NMR analysis.

In a typical procedure tosyloxime $\mathbf{4}$ was solubilized in benzene mixed with potassium carbonate (10 equiv), triethylamine ( 0.3 equiv), and a 1,3-diene and stirred for four days at room temperature. The primary cycloadducts 6a,b,d,e were obtained in 9-59\% yield. Derivative 7 was obtained in the case of reaction with the Danishefsky diene in $39 \%$ yield, by rearrangement of the primary cycloadduct 6c (Scheme 2). In case 6f, the silyl group cleaved during chromatography giving $\mathbf{8}$.

The moderate yields are certainly the reflection of the two-step sequence in the one-pot procedure together with the instability of the azirine because of the presence of the two electron-withdrawing substituents in the ring.

Cycloadduct $\mathbf{6 a}$, obtained from reaction of azirine $\mathbf{5}$ with 2,3-dimethylbuta-1,3-diene, was isolated in very low yield, even in the presence of a large excess of diene $(5$ equiv). Difficulties of the same type had been reported by Davis in reaction of a $2 \mathrm{H}$-azirine-3-phosphonate with 2,3dimethylbuta-1,3-diene, where 100 equivalents of the diene were required. ${ }^{9}$

Reaction of the azirine 5 with 1-methoxybuta-1,3-diene evidenced that the regioselectivity of the cycloaddition is governed by electronic effects. ${ }^{1} \mathrm{H}$ and ${ }^{13} \mathrm{C}$ NMR data of product $\mathbf{6 b}$ are in accordance with the electron-withdrawing effect of the two heteroatoms attached to $\mathrm{C} 2 ; \mathrm{H} 2$ is at $\delta_{\mathrm{H}}=4.80$ and $\mathrm{C} 2$ at $\delta_{\mathrm{C}}=85.6$. The cycloaddition products were obtained as single isomers, presumably formed by endo-selective processes, as generally observed in reactions of $2 \mathrm{H}$-azirines with 1,3 -dienes. ${ }^{8}$ Furan and their derivatives are exceptions due to retro-Diels-Alder cycloadditions of the initially formed endo-cycloadduct that isomerize to the exo-products. ${ }^{11 \mathrm{~b}}$ The low-field resonance of $\mathrm{H} 3$ in the tricyclic products obtained by reaction of $2 \mathrm{H}$-azirines with cyclopentadiene is a clear feature of the endo selectivity. ${ }^{8}$ This can be ascribed to the anisotropy of the backside double bond over H3, due to constraints of the tricyclic structure. The chemical shift value of $\mathrm{H} 3$ in compound $\mathbf{6 d}$ correspond to such an effect appearing at $\delta_{\mathrm{H}}=1.62$.

Features of pyridinone 7 , obtained by rearrangement of 6c, are the two hydrogens of the $\mathrm{CH}_{2}$ group, coupling to the phosphorus nucleus with $J=12.0 \mathrm{~Hz}$; the signal at $\delta_{\mathrm{H}}=6.45$, assigned to $\mathrm{H} 5$, shows a doublet of doublets $\left({ }^{3} J=3.0 \mathrm{~Hz}\right.$ to $\mathrm{H} 3$ and ${ }^{2} J=7.6 \mathrm{~Hz}$ to $\left.\mathrm{H} 6\right)$ and two doublets at $\delta_{\mathrm{H}}=7.35$, corresponding to $\mathrm{H} 6$, and at $\delta_{\mathrm{H}}=7.03$, corresponding to $\mathrm{H} 3$, show matching couplings to $\mathrm{H} 5$. Rearrangements of this type have been noticed before in bicyclic adducts obtained from the Danishefsky diene and $2 \mathrm{H}$-azirines bearing electrophilic groups. ${ }^{16}$

In summary, cycloaddition reactions of ethyl 2-(diisopropoxyphosphoryl)-2H-azirine-3-carboxylate to nucleophilic dienes produced, in moderate yields, a number of functionalized six-membered-ring fused aziridines. These may eventually be valuable intermediates at the synthesis of interesting biological compounds related to $(S)$-AP3. Studies to improve the reaction efficiency as well as the<smiles>CCOC(=O)C1(C(=O)OCC)C2CCC(C2)N1P(=O)(OCC)OCC</smiles>

6e $20 \%$

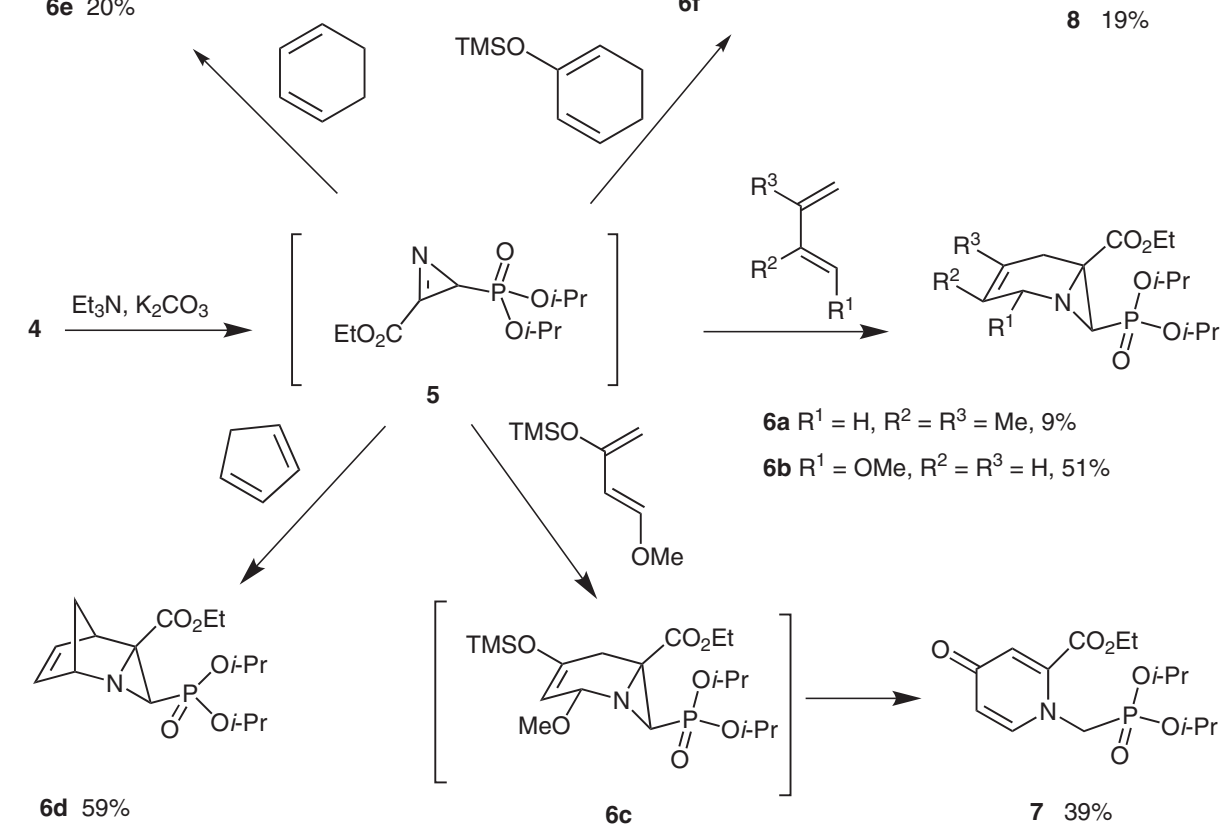

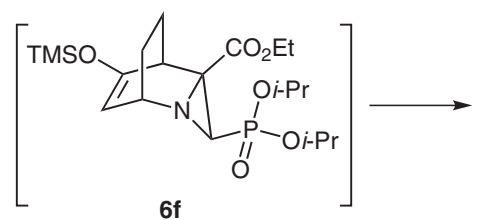

$6 f$

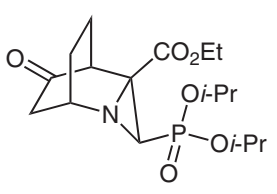

$819 \%$

Scheme 2 Generation of azirine $\mathbf{5}$ and its cycloaddition reactions with 1,3-dienes 
development of an asymmetric synthesis or generation of the azirine are ongoing.

${ }^{1} \mathrm{H}$ and ${ }^{13} \mathrm{C}$ NMR spectra (100.6 or $75.5 \mathrm{MHz}$ ) were recorded on a Bruker Avance III $400(400 \mathrm{MHz})$ spectrometer or on a Bruker WM AMX (300 MHz), using TMS as internal standard. IR spectra were recorded on a Perkin-Elmer 1640-FT spectrophotometer. Samples were run as thin films. Mass spectra were recorded on a VG Autospec M. Purification of crude samples was performed by dry flash chromatography, using silica gel purchased from Carlo Erba (35-70 $\mathrm{m} \mu)$.

\section{Ethyl 3-(Diisopropoxyphosphoryl)-2-(hydroxyimino)pro- panoate (3)}

To ethyl bromopyruvate oxime $(4.7 \mathrm{~g}, 22 \mathrm{mmol})$ dissolved in $\mathrm{CH}_{2} \mathrm{Cl}_{2}(30 \mathrm{~mL})$ was added $\mathrm{P}(\mathrm{O} i-\mathrm{Pr})_{3}(6 \mathrm{~mL}, 24 \mathrm{mmol})$ and the mixture stirred at $35^{\circ} \mathrm{C}$ for $16 \mathrm{~h} . \mathrm{H}_{2} \mathrm{O}(30 \mathrm{~mL})$ was added and the mixture stirred at r.t. for a further $30 \mathrm{~min}$ and the organic phase was dried $\left(\mathrm{MgSO}_{4}\right)$ and evaporated under vacuum. The oily residue was subjected to dry-flash chromatography (silica gel, $\mathrm{CH}_{2} \mathrm{Cl}_{2}-\mathrm{EtOAc}$, $10: 1)$, affording $\mathbf{3}(5.13 \mathrm{~g}, 79 \%)$ as a as colorless oil.

IR(neat): 3167, 2982, 2936, 1720, 1252, $995 \mathrm{~cm}^{-1}$.

${ }^{1} \mathrm{H}$ NMR $\left(300 \mathrm{MHz}, \mathrm{CDCl}_{3}\right): \delta=1.22-1.30(\mathrm{~m}, 15 \mathrm{H}, 5 \mathrm{Me}), 3.29$ $\left(\mathrm{d},{ }^{1} J_{\mathrm{PH}}=24.0 \mathrm{~Hz}, 2 \mathrm{H}\right), 4.19-4.29\left(\mathrm{~m}, 2 \mathrm{H}, \mathrm{OCH}_{2}\right), 4.66-4.74(\mathrm{~m}$, $2 \mathrm{H}, 2 \mathrm{OCH})$.

${ }^{13} \mathrm{C}$ NMR (75.47 MHz, $\left.\mathrm{CDCl}_{3}\right): \delta=14.1(\mathrm{Me}), 23.6(\mathrm{Me}), 23.7$ $(\mathrm{Me}), 24.6(\mathrm{Me}), 24.6\left(\mathrm{~d},{ }^{1} J_{\mathrm{PC}}=83.8 \mathrm{~Hz}\right), 61.5\left(\mathrm{OCH}_{2}\right), \sim 71.1(\mathrm{~d}$, $\left.{ }^{2} J_{\mathrm{PC}}=13.6 \mathrm{~Hz}, \mathrm{OCH}\right), 143.6(\mathrm{C}=\mathrm{N}), 163.9(\mathrm{CO})$.

HRMS (ESI-TOF): $m / z[\mathrm{M}+\mathrm{H}]^{+}$calcd for $\mathrm{C}_{11} \mathrm{H}_{23} \mathrm{NO}_{6} \mathrm{P}: 296.1263$; found: 296.1258 .

\section{Ethyl 3-(Diisopropoxyphosphoryl)-2-[(tosyloxy)imino]pro-} panoate (4)

To a soln of $\mathbf{3}(4.5 \mathrm{~g}, 15 \mathrm{mmol})$ in $\mathrm{CH}_{2} \mathrm{Cl}_{2}(40 \mathrm{~mL})$ was added $\mathrm{Na}_{2} \mathrm{CO}_{3}(4.8 \mathrm{~g}, 45 \mathrm{mmol})$ followed by $\mathrm{TsCl}(3.24 \mathrm{~g}, 17 \mathrm{mmol})$ and the mixture stirred until the disappearance of the starting oxime ( 4 h). The insolubles were removed by filtration and the solvent was evaporated to afford a residue that was subjected to dry-flash chromatography (silica gel, hexanes- $\mathrm{CH}_{2} \mathrm{Cl}_{2}-\mathrm{EtOAc}$, increasing polarity) to product $4(4.2 \mathrm{~g}, 62 \%)$ as a pale yellow thick oil.

IR(neat): 2983, 1737, 1386, 1267, 1194, $993 \mathrm{~cm}^{-1}$.

${ }^{1} \mathrm{H}$ NMR $\left(300 \mathrm{MHz}, \mathrm{CDCl}_{3}\right): \delta=1.19-1.30(\mathrm{~m}, 15 \mathrm{H}, 5 \mathrm{Me}), 2.38$ (s, $3 \mathrm{H}, \mathrm{PhMe}), 3.25\left(\mathrm{~d},{ }^{1} J_{\mathrm{PH}}=24.0 \mathrm{~Hz}, 2 \mathrm{H}\right), 4.23(\mathrm{q}, J=6.0 \mathrm{~Hz}, 2$ $\left.\mathrm{H}, \mathrm{OCH}_{2}\right), 4.57-4.64(\mathrm{~m}, 2 \mathrm{H}, 2 \mathrm{OCH}), 7.28(\mathrm{~d}, J=6.0 \mathrm{~Hz}, 2 \mathrm{H})$, $7.83(\mathrm{~d}, J=6.0 \mathrm{~Hz}, 2 \mathrm{H})$.

${ }^{13} \mathrm{C} \mathrm{NMR}$ (75.5 MHz, $\mathrm{CDCl}_{3}$ ): $\delta=13.8(\mathrm{Me}), 21.6(\mathrm{Me}), 23.4(\mathrm{Me})$, $23.5(\mathrm{Me}), 23.6(\mathrm{Me}), 23.7(\mathrm{Me}), 26.5\left(\mathrm{~d},{ }^{1} J_{\mathrm{PC}}=135.8 \mathrm{~Hz}, \mathrm{PCH}_{2}\right)$, $62.8(\mathrm{OCH}), \sim 72.0\left(\mathrm{~d},{ }^{2} J_{\mathrm{PC}}=12.1 \mathrm{~Hz}, \mathrm{OCH}\right), 131.7(\mathrm{C}, \mathrm{Ar}), 145.7$ $(\mathrm{C}, \mathrm{Ar}), 152.1(\mathrm{C}=\mathrm{N}), 161.1(\mathrm{CO})$.

HRMS (ESI-TOF): $m / z[\mathrm{M}+\mathrm{H}]^{+}$calcd for $\mathrm{C}_{18} \mathrm{H}_{29} \mathrm{NO}_{8} \mathrm{PS}$ : 450.1351; found: 450.1346 .

\section{Cycloaddition Reactions; General Procedure}

To a soln of tosyloxime $4(0.3 \mathrm{~g}, 0.69 \mathrm{mmol})$ in benzene $(5 \mathrm{~mL})$ was added $\mathrm{Et}_{3} \mathrm{~N}$ (30 $\mu \mathrm{L}, 0.33$ equiv), $\mathrm{K}_{2} \mathrm{CO}_{3}(0.96 \mathrm{~g}, 6.9 \mathrm{mmol}, 10$ equiv), and the diene (1.0 equiv to large excess). [Cyclopentadiene was used in large excess $(1 \mathrm{~mL})$, other dienes were used in excess: the Danishefsky diene (1.5 equiv), 1-methoxybuta-1,3-diene (2 equiv), 2,3-dimethylbuta-1,3-diene (1.5 equiv or 5 equiv).] The mixture was stirred at r.t. for 4 d. Evaporation of the solvent gave the crude product, which was subjected to dry-flash chromatography (silica gel, petroleum ether- $\mathrm{Et}_{2} \mathrm{O}$, polarity gradient or $\mathrm{EtOAc}-$ $\mathrm{MeOH}, 3: 1$ for 7) affording products $\mathbf{6 - 8}$ as oils.
Ethyl 7-(Diisopropoxyphosphoryl)-3,4-dimethyl-1-azabicyclo[4.1.0]hept-3-ene-6-carboxylate (6a)

(i) 2,3-Dimethybuta-1,3-diene (1.5 equiv); yield: $0.020 \mathrm{~g} \mathrm{(8 \% ).}$

(ii) 2,3-Dimethybuta-1,3-diene (5.0 equiv); yield: $0.025 \mathrm{~g}$ (9\%).

IR (neat): 3458, 2981, 2933, $1753 \mathrm{~cm}^{-1}$.

${ }^{1} \mathrm{H}$ NMR $\left(300 \mathrm{MHz}, \mathrm{CDCl}_{3}\right): \delta=1.29-1.33(\mathrm{~m}, 15 \mathrm{H}, 5 \mathrm{Me}), 1.52$ (br s, $3 \mathrm{H}, \mathrm{Me}$ ), 1.63 (br s, $3 \mathrm{H}, \mathrm{Me}$ ), 2.16 (d, $J=17.3 \mathrm{~Hz}, 1 \mathrm{H}, \mathrm{H} 7$ ), 2.38 (br d, $J=17.3 \mathrm{~Hz}, 1 \mathrm{H}, \mathrm{H} 5), 2.70$ (d, $J=17.6 \mathrm{~Hz}, 1 \mathrm{H}, \mathrm{H} 5)$, $3.23(\mathrm{~d}, J=17.0 \mathrm{~Hz}, 1 \mathrm{H}, \mathrm{H} 2), 3.73(\mathrm{~d}, J=17.0 \mathrm{~Hz}, 1 \mathrm{H}, \mathrm{H} 2), 4.16$ $4.26\left(\mathrm{~m}, 2 \mathrm{H}, \mathrm{OCH}_{2}\right), 4.68-4.80(\mathrm{~m}, 2 \mathrm{H}, 2 \mathrm{OCH})$.

${ }^{13} \mathrm{C} \mathrm{NMR}\left(75.5 \mathrm{MHz}, \mathrm{CDCl}_{3}\right): \delta=13.1(\mathrm{Me}), 15.4(\mathrm{Me}), 17.5(\mathrm{Me})$, $22.6(\mathrm{Me}), 22.9(\mathrm{Me}), 23.95(\mathrm{Me}), 23.05(\mathrm{Me}), 23.1(\mathrm{Me}), 28.4(\mathrm{~d}$, $\left.{ }^{3} J_{\mathrm{PC}}=19 \mathrm{~Hz}, \mathrm{CH}_{2}, \mathrm{C} 5\right), 33.1\left(\mathrm{~d},{ }^{1} J_{\mathrm{PC}}=216 \mathrm{~Hz}, \mathrm{C} 7\right), 45.0(\mathrm{~d}$, $\left.{ }^{2} J_{\mathrm{PC}}=5.0 \mathrm{~Hz}, \mathrm{C} 6\right), 51.6\left(\mathrm{~d},{ }^{3} J_{\mathrm{PC}}=6.6 \mathrm{~Hz}, \mathrm{CH}_{2}, \mathrm{C} 2\right), 60.3\left(\mathrm{CH}_{2} \mathrm{O}\right)$, $69.8\left(\mathrm{~d},{ }^{3} J_{\mathrm{PC}}=6.0 \mathrm{~Hz}, \mathrm{COH}\right), 69.9\left(\mathrm{~d},{ }^{3} J_{\mathrm{PC}}=6.3 \mathrm{~Hz}, \mathrm{COH}\right), 118.7$ (C3 or C4), 119.1 (C4 or C3), 168.7 (CO).

HRMS (FAB): $m / z[\mathrm{M}+\mathrm{H}]^{+}$calcd for $\mathrm{C}_{17} \mathrm{H}_{31} \mathrm{NO}_{5} \mathrm{P}: 360.1940$; found: 360.1927 .

\section{Ethyl 7-(Diisopropoxyphosphoryl)-2-methoxy-1-azabicyc-} lo[4.1.0]hept-3-ene-6-carboxylate $(6 \mathrm{~b})$

Yield: $0.125 \mathrm{~g}(51 \%)$.

IR (neat): 3467, 2980, 2931, 1754, $1731 \mathrm{~cm}^{-1}$.

${ }^{1} \mathrm{H} \mathrm{NMR}\left(300 \mathrm{MHz}, \mathrm{CDCl}_{3}\right): \delta=1.23-1.28(\mathrm{~m}, 15 \mathrm{H}, 5 \mathrm{Me}), 2.30$ $(\mathrm{d}, J=16.6 \mathrm{~Hz}, 2 \mathrm{H}, \mathrm{H} 5, \mathrm{H} 7), 2.78(\mathrm{dd}, J=6.1,18.5 \mathrm{~Hz}, 1 \mathrm{H}, \mathrm{H} 5)$, 3.61 (s, $3 \mathrm{H}, \mathrm{OMe}$ ), 4.16-4.18 (m, $2 \mathrm{H}, \mathrm{OCH}_{2}$ ), 4.68 (br s, $2 \mathrm{H}$, $2 \mathrm{OCH}), 4.80(\mathrm{~s}, 1 \mathrm{H}, \mathrm{H} 2), 5.39(\mathrm{~d}, J=10.0 \mathrm{~Hz}, 1 \mathrm{H}, \mathrm{H} 3), 5.62-5.63$ (m, $1 \mathrm{H}, \mathrm{H} 4)$.

${ }^{13} \mathrm{C} \mathrm{NMR}\left(75.5 \mathrm{MHz}, \mathrm{CDCl}_{3}\right): \delta=14.4(\mathrm{Me}), 24.1(\mathrm{Me}), 24.2\left(\mathrm{CH}_{2}\right.$, C5), $24.4(\mathrm{Me}), 24.42(\mathrm{Me}), 24.6(\mathrm{Me}), 33.2\left(\mathrm{~d},{ }^{1} J_{\mathrm{PC}}=217 \mathrm{~Hz}, \mathrm{C} 7\right)$, $45.6\left(\mathrm{~d},{ }^{2} J_{\mathrm{PC}}=4.5 \mathrm{~Hz}, \mathrm{C} 6\right), 57.3(\mathrm{OMe}), 61.8\left(\mathrm{OCH}_{2}\right), 71.3(\mathrm{~d}$, $\left.{ }^{2} J_{\mathrm{PC}}=6.8 \mathrm{~Hz}\right) 71.5\left(\mathrm{~d},{ }^{2} J_{\mathrm{PC}}=6.8 \mathrm{~Hz}, \mathrm{OCH}\right), 85.6(\mathrm{C} 2), 123.1(\mathrm{C} 3$ or C4), 124.5 (C4 or C3), $168.0(\mathrm{CO})$.

HRMS (FAB): $m / z[\mathrm{M}+\mathrm{H}]^{+}$calcd for $\mathrm{C}_{16} \mathrm{H}_{29} \mathrm{NO}_{6} \mathrm{P}: 362.1732$; found: 362.1731 .

Ethyl 3-(Diisopropoxyphosphoryl)-2-azatricyclo[3.2.1.0 $\left.0^{2,4}\right]$ oct6-ene-4-carboxylate (6d)

Yield: $0.140 \mathrm{~g}(59 \%)$.

IR (neat): 3467, 2981, 2937, $1741 \mathrm{~cm}^{-1}$.

${ }^{1} \mathrm{H}$ NMR $\left(300 \mathrm{MHz}, \mathrm{CDCl}_{3}\right): \delta=1.27-1.34(\mathrm{~m}, 15 \mathrm{H}, 5 \mathrm{Me}), 1.62$ $(\mathrm{d}, J=12.5 \mathrm{~Hz}, 1 \mathrm{H}, \mathrm{H} 3), 1.71(\mathrm{t}, J=8.6 \mathrm{~Hz}, 1 \mathrm{H}, \mathrm{H} 8), 2.43(\mathrm{~d}$, $J=8.6 \mathrm{~Hz}, 1 \mathrm{H}, \mathrm{H} 8), 3.29$ (br s, $1 \mathrm{H}, \mathrm{H} 5), 4.24-4.29$ (m, $3 \mathrm{H}, \mathrm{OCH}_{2}$, $\mathrm{H} 1)$, 4.67-4.78 (m, $2 \mathrm{H}, 2 \mathrm{OCH}), 5.71-5.73(\mathrm{~m}, 1 \mathrm{H}, \mathrm{H} 6$ or H7), 6.18-6.22 (m, $1 \mathrm{H}, \mathrm{H} 7$ or H6).

${ }^{13} \mathrm{C}$ NMR (75.5 MHz, $\left.\mathrm{CDCl}_{3}\right): \delta=14.5(\mathrm{Me}), 24.3(\mathrm{Me}), 24.4(\mathrm{Me})$, $24.43(\mathrm{Me}), 24.5(\mathrm{Me}), 45.8\left(\mathrm{~d},{ }^{1} J_{\mathrm{PC}}=205 \mathrm{~Hz}, \mathrm{C} 3\right), \sim 48(\mathrm{C} 6), 49.6$ (C5), $59.4(\mathrm{~d}, J=3.0 \mathrm{~Hz}, \mathrm{C} 8), 62.0\left(\mathrm{OCH}_{2}\right), 67.6\left(\mathrm{~d},{ }^{3} J_{\mathrm{PC}}=7.5 \mathrm{~Hz}\right.$, $\mathrm{C} 1), 71.3\left(\mathrm{~d},{ }^{2} J_{\mathrm{PC}}=6.8 \mathrm{~Hz}, \mathrm{OCH}\right), 71.5\left(\mathrm{~d},{ }^{2} J_{\mathrm{PC}}=6.8 \mathrm{~Hz}, \mathrm{OCH}\right)$, 128.8 (C5 or C6), 133.3 (C6 or C5), 172.0 (CO).

HRMS (FAB): $m / z[\mathrm{M}+\mathrm{H}]^{+}$calcd for $\mathrm{C}_{16} \mathrm{H}_{27} \mathrm{NO}_{5} \mathrm{P}: 344.1627$; found: 344.1615 .

Ethyl 3-(Diisopropoxyphosphoryl)-2-azatricyclo[3.2.2.0 $\left.{ }^{2,4}\right]$ non6-ene-4-carboxylate (6e)

Yield: $0.088 \mathrm{~g}(20 \%)$.

IR (neat): 2933, 1749, 1653, $1281 \mathrm{~cm}^{-1}$.

${ }^{1} \mathrm{H}$ NMR (400 MHz, $\left.\mathrm{CDCl}_{3}\right): \delta=1.10(\mathrm{~m}, 1 \mathrm{H}, \mathrm{H} 8$ or H9), 1.24 1.34 (m, 16 H, $5 \mathrm{Me}, 1 \mathrm{H}, \mathrm{H} 9$ or H8), $1.52(\mathrm{~d}, J=13.2 \mathrm{~Hz}, 1 \mathrm{H}, \mathrm{H} 3)$, 1.69 (m, $1 \mathrm{H}, \mathrm{H} 8$ or H9), 2.1 (m, $1 \mathrm{H}, \mathrm{H} 9$ or H8), 3.12 (m, $1 \mathrm{H}, \mathrm{H} 5)$, $3.99(\mathrm{~m}, 1 \mathrm{H}, \mathrm{H1}), 4.26\left(\mathrm{~m}, 2 \mathrm{H}, \mathrm{CO}_{2} \mathrm{CH}_{2} \mathrm{CH}_{3}\right), 4.66-4.81(\mathrm{~m}, 2 \mathrm{H}$, $2 \mathrm{OCH}), 5.68$ (m, $1 \mathrm{H}, \mathrm{H} 6$ or H7), 6.22 (m, $1 \mathrm{H}, \mathrm{H} 7$ or H6). 
${ }^{13} \mathrm{C}$ NMR $\left(100.6 \mathrm{MHz}, \mathrm{CDCl}_{3}\right): \delta=14.1\left(\mathrm{CO}_{2} \mathrm{CH}_{2} \mathrm{CH}_{3}\right), 19.8(\mathrm{C} 8$ or C9), 23.6 (C9 or C8), 23.82, 23.9, 24.0, 24.0 (4 Me), 31.0 (d, $\left.{ }^{1} J_{\mathrm{PC}}=212.5 \mathrm{~Hz}, \mathrm{C} 3\right), 32.9(\mathrm{~d}, J=2.6 \mathrm{~Hz}, \mathrm{C} 5), 52.3\left(\mathrm{~d},{ }^{3} J_{\mathrm{PC}}=9.2\right.$ $\mathrm{Hz}, \mathrm{C} 1), 61.4\left(\mathrm{CO}_{2} \mathrm{CH}_{2} \mathrm{CH}_{3}\right), 70.7\left(\mathrm{~d},{ }^{2} J_{\mathrm{PC}}=6.3 \mathrm{~Hz}, \mathrm{OCH}\right), 71.0(\mathrm{~d}$, $\left.{ }^{2} J_{\mathrm{PC}}=6.3 \mathrm{~Hz}, \mathrm{OCH}\right), 125.1(\mathrm{C} 6$ or C7), 130.1 (C7 or C6), 125.5 (q, $\mathrm{C} 2), 169.2(\mathrm{C}=\mathrm{O})$.

HRMS (FAB): $m / z,[\mathrm{M}+\mathrm{H}]^{+}$calcd for $\mathrm{C}_{17} \mathrm{H}_{29} \mathrm{NO}_{5} \mathrm{P}: 358.1783$; found: 358.1784 .

Ethyl 1-[(Diisopropoxyphosphoryl)methyl]-4-oxo-1,4-dihydropyridine-2-carboxylate (7)

Yield: $0.092 \mathrm{~g}(39 \%)$.

IR (neat): 3440, 2983, 2938, 1733, 1633, $1573 \mathrm{~cm}^{-1}$.

${ }^{1} \mathrm{H}$ NMR $\left(300 \mathrm{MHz}, \mathrm{CDCl}_{3}\right): \delta=1.21-1.27(\mathrm{~m}, 12 \mathrm{H}, 4 \mathrm{Me}), 1.36$ $(\mathrm{t}, J=7.2 \mathrm{~Hz}, 3 \mathrm{H}, \mathrm{Me}), 3.73\left(\mathrm{q}, J=7.2 \mathrm{~Hz}, 2 \mathrm{H}, \mathrm{OCH}_{2}\right), 4.63-4.71$ $(\mathrm{m}, 2 \mathrm{H}, 2 \mathrm{OCH}), 4.71\left(\mathrm{~d}, J=12.0 \mathrm{~Hz}, 2 \mathrm{H}, \mathrm{H1}{ }^{\prime}\right), 6.45(\mathrm{dd}, J=3.0$, $7.6 \mathrm{~Hz}, 1 \mathrm{H}, \mathrm{H} 5), 7.03(\mathrm{~d}, J=3.0 \mathrm{~Hz}, 1 \mathrm{H}, \mathrm{H} 3), 7.35(\mathrm{~d}, J=7.6 \mathrm{~Hz}$, $1 \mathrm{H}, \mathrm{H} 6)$.

${ }^{13} \mathrm{C}$ NMR (75.5 MHz, $\mathrm{CDCl}_{3}$ ): $\delta=14.3(\mathrm{Me}), 24.21(\mathrm{Me}), 24.27$ (Me), $24.32(\mathrm{Me}), 49.7\left(\mathrm{~d},{ }^{1} \mathrm{~J}_{\mathrm{PC}}=156 \mathrm{~Hz}, \mathrm{Cl}^{\prime}, \mathrm{CH}_{2}\right), 63.3\left(\mathrm{CH}_{2} \mathrm{O}\right)$, $73.0\left(\mathrm{~d},{ }^{2} J_{\mathrm{PC}}=7.5 \mathrm{~Hz}, \mathrm{OCH}\right), 119.7(\mathrm{C} 5), 123.3(\mathrm{C} 3), 140.2(\mathrm{C} 2)$, $162.9(\mathrm{CO}), 179.5(\mathrm{CO})$.

HRMS (FAB): $m / z[\mathrm{M}+\mathrm{H}]^{+}$calcd for $\mathrm{C}_{15} \mathrm{H}_{25} \mathrm{NO}_{6} \mathrm{P}: 346.1419$; found: 346.1419 .

\section{Ethyl 3-(Diisopropoxyphosphoryl)-6-oxo-2-azatricyc-}

lo[3.2.2.0 $\left.{ }^{2,4}\right]$ nonane-4-carboxylate $(8)$

Yield: $0.077 \mathrm{~g}(19 \%)$.

IR (neat): 3459, 3274, 3054, 1734, $1602 \mathrm{~cm}^{-1}$.

${ }^{1} \mathrm{H}$ NMR (400 MHz, $\left.\mathrm{CDCl}_{3}\right): \delta=1.29-1.30(\mathrm{~m}, 15 \mathrm{H}, 5 \mathrm{Me}), 1.73-$ $1.80(\mathrm{~m}, 2 \mathrm{H}), 1.88(\mathrm{~d}, J=9.3 \mathrm{~Hz}, 1 \mathrm{H}, \mathrm{H} 3), 2.07-2.32(\mathrm{~m}, 4 \mathrm{H})$, 3.07 (br s, $1 \mathrm{H}, \mathrm{H} 1), 3.79$ (br s, $1 \mathrm{H}, \mathrm{H} 5), 4.26\left(\mathrm{~m}, 2 \mathrm{H}, \mathrm{CH}_{2} \mathrm{CH}_{3}\right.$ ), 4.75 (m, $2 \mathrm{H}, 2 \mathrm{OCH})$.

${ }^{13} \mathrm{C}$ NMR $\left(100.6 \mathrm{MHz}, \mathrm{CDCl}_{3}\right): \delta=14.0(\mathrm{Me}), 18.5$ (C8 or C9), $23.8(\mathrm{Me}), 23.92(\mathrm{Me}), 23.96(\mathrm{Me}), 24.0(\mathrm{Me}), 24.6\left(\mathrm{CH}_{2}\right), 33.5(\mathrm{~d}$, $\left.{ }^{1} J_{\mathrm{PC}}=213.3 \mathrm{~Hz}, \mathrm{C} 3\right), 39.6\left(\mathrm{C} 8\right.$ or C9), $43.7\left(\mathrm{~d},{ }^{3} J_{\mathrm{PC}}=2.0 \mathrm{~Hz}, \mathrm{C} 5\right)$, $45.8\left(\mathrm{~d},{ }^{2} J_{\mathrm{PC}}=4.0 \mathrm{~Hz}, \mathrm{C} 4\right), 49.7\left(\mathrm{~d},{ }^{3} J_{\mathrm{PC}}=8.0 \mathrm{~Hz}, \mathrm{C} 1\right), 61.8(\mathrm{OCH})$, $71.4\left(\mathrm{~d},{ }^{2} J_{\mathrm{PC}}=6.0 \mathrm{~Hz}, \mathrm{OCH}\right), 167.0(\mathrm{C}=\mathrm{O}), 207.6(\mathrm{C}=\mathrm{O})$.

HRMS (FAB): $m / z[M+\mathrm{H}]^{+}$calcd for $\mathrm{C}_{17} \mathrm{H}_{29} \mathrm{NO}_{6} \mathrm{P}: 374.1732$; found: 374.1729 .

\section{Acknowledgment}

Thanks are due to Fundação para a Ciência e Tecnologia and Xunta de Galicia under project 07CSA008203PR, for financial support. NMR spectrometer Bruker Avance II 400, acquired with funds from FCT and FEDER (part of the National NMR Network).

\section{References}

(1) For reviews, see: (a) Palacios, F.; Ochoa de Retana, A. M.; Marigorta, E. M.; de los Santos, J. M. Org. Prep. Proced. Int. 2002, 34, 219. (b) Gilchrist, T. L. Aldrichimica Acta 2001, 34, 51. (c) Palacios, F.; Ochoa de Retana, A. M.; Marigorta, E. M.; de los Santos, J. M. Eur. J. Org. Chem. 2001, 2401.
(2) (a) Pinho e Melo, T. M. V. D.; Lopes, C. S. J.; Gonçalves, A. M. d' A. R.; Beja, J. A.; Paixão, A. M.; Silva, M. R.; Alte da Veiga, L. J. Org. Chem. 2002, 67, 66. (b) Davis, F. A.; Liang, C.; Liu, H. J. Org. Chem. 1997, 62, 3796. (c) Banert, K.; Kohler, F. Angew. Chem. Int. Ed. 2001, 40, 174.

(3) Heimgartner, H. Angew. Chem., Int. Ed. Engl. 1991, 30, 238.

(4) Miller, T. W.; Tristram, E. W.; Wolf, F. J. J. Antibiot. 1971, $24,48$.

(5) Molinski, T. F.; Ireland, C. M. J. Org. Chem. 1988, 53, 2103.

(6) (a) Tanner, D. Angew. Chem., Int. Ed. Engl. 1994, 33, 599. (b) Filigheddu, S. N.; Taddei, M. Tetrahedron Lett. 1998, 39, 3857. (c) Zwanenburg, B.; Thi, L. J. Pure Appl. Chem. 1996, 68, 735. (d) Davis, F. A.; Liu, H.; Reddy, C. V. Tetrahedron Lett. 1996, 37, 5473.

(7) (a) Righi, G.; D’ Achielle, R. Tetrahedron Lett. 1996, 37, 6893. (b) Lim, Y.; Lee, W. K. Tetrahedron Lett. 1995, 36, 8431. (c) Tanner, D.; Bergsson, C.; Dhaliwal, H. K. Tetrahedron Lett. 1990, 31, 1903.

(8) Alves, M. J.; Gilchrist, T. L. J. Chem. Soc., Perkin Trans. 1 1998, 299.

(9) (a) Davis, F. A.; Wu, Y.; Yan, H.; Prasad, K. R.; McCoull, W. Org. Lett. 2002, 4, 655. (b) Bickley, J. F.; Gilchrist, T. L.; Mendonça, R. ARKIVOC 2002, (vi), 192.

(10) Alves, M. J.; Gil Fortes, A.; Lemos, A.; Martins, C. Synthesis 2005, 555.

(11) (a) Nair, V. J. J. Org. Chem. 1972, 37, 2508. (b) Hassner, A.; Anderson, D. J. J. Org. Chem. 1974, 39, 2031. (c) Ray, C. A.; Risberg, E.; Somfai, P. Tetrahedron Lett. 2001, 42, 9289. (d) Ray, C. A.; Risberg, E.; Somfai, P. Tetrahedron 2002, 58, 5983.

(12) (a) Young, A. B.; Greenamyre, J. T.; Hollingsworth, Z.; Albin, R.; D'Amato, C.; Shoulson, I.; Penny, J. B. Science 1988, 241, 981. (b) Reyes-Rangel, G.; Marañón, V.; AvilaOrtiz, C. G.; Parrodi, C. A.; Quintero, L.; Juaristi, E. Tetrahedron 2006, 62, 8404.

(13) (a) Engel, R. In Handbook of Organophosphorus Chemistry; Dekker Inc.: New York, 1992. (b) Kafarski, P.; Lejczak, B. Phosphorus, Sulfur Silicon Relat. Elem. 1991, 63, 193.

(14) (a) Gilchrist, T. L.; Roberts, T. G. J. Chem. Soc., Perkin Trans. 1 1983, 1283. (b) Ottenheijm, H. C. J.; Plate, R.; Noordik, J. H.; Herscheid, J. D. M. J. Org. Chem. 1982, 47, 2147.

(15) (a) Palacios, F.; Ochoa de Retana, A. M.; Gil, J. I.; Ezpeleta, J. M. J. Org. Chem. 2000, 65, 3213. (b) Palacios, F.; Ochoa de Retana, A. M.; Gil, J. I. Tetrahedron Lett. 2000, 41, 5363. (c) Palacios, F.; Ochoa de Retana, A. M.; Gil, J. I.; López de Munain, R. Org. Lett. 2002, 4, 2405. (d) Palacios, F.; Ochoa de Retana, A. M.; Gil, J. I.; Alonso, J. M. Tetrahedron: Asymmetry 2002, 13, 2525. (e) Palacios, F.; Aparicio, D.; Ochoa de Retana, A. M.; de los Santos, J. M.; Gil, J. I.; López de Munain, R. Tetrahedron: Asymmetry 2003, 14, 689. (f) Palacios, F.; Ochoa de Retana, A. M.; Gil, J. I.; Alonso, J. M. Tetrahedron 2004, 60, 8937.

(16) Alves, M. J.; Gil Fortes, A.; Costa, F. T.; Duarte, V. C. M. Tetrahedron 2007, 63, 11167. 